\title{
近红外发光的稀土分子探针在生物成像中的 设计及应用
}

\author{
朱晓飞 ${ }^{1,2 \dagger}$, 金国庆 ${ }^{1 \dagger}$, 张俊龙 $^{1^{*}}$ \\ 1. 北京大学化学与分子工程学院, 北京 100871 \\ 2. 长春工业大学化学与生命科学学院, 长春 130012 \\ †同等贡献 \\ *通讯作者, E-mail: zhangjunlong@pku.edu.cn
}

收稿日期：2020-07-03; 接受日期：2020-08-04; 网络版发表日期：2020-10-26

国家重点基础研究发展计划(编号: 2015CB856301)、国家自然科学基金(编号: 21571007, 21621061, 21778002, 21861162008)、吉林省教育厅 项目(JJKH20200647KJ)和吉林省科技厅项目(20200403154SF)资助

\begin{abstract}
摘要近红外生物成像是一种实时监测各种生理和病理过程、实现生物实体可视化的前沿技术，具有背景干 扰少、时空分辨率高、组织穿透能力强等优点，在临床成像研究和应用方面具有巨大潜力，受到日益广泛的关 注。其中, 近红外发光分子探针的设计、性质及应用至关重要, 已经成为决定近红外生物成像领域发展的关键因 素. 稀土配合物具有小尺寸、低毒性、长寿命、大Stokes位移、抗光漂白、独特的线状发射光谱(元素特征的f-f 跃迁)等优点, 成为新兴的近红外发光探针, 展现出极好的生物医学应用前景. 本文综述了近红外稀土分子探针的 设计原理和研究进展, 结合近年来其在生物医学方面的应用, 展望了其发展的方向和趋势以及对稀土化学生物学 发展的推动作用.
\end{abstract}

关键词近红外稀土配合物, 荧光探针, 生物成像, 生物传感, 稀土化学生物学

\section{1 引言}

生物组织在近红外(NIR，700 1700 nm)光谱区域 具有较低的消光系数, 有利于减少生物实体与近红外 光光子之间的相互作用，从而降低生物实体对近红外 光的吸收、散射以及生物组织的自发荧光，大大提高 生物组织成像深度和信噪比等，因此近红外生物成像 在临床前成像研究和治疗中受到了广泛的关注 ${ }^{[1 \sim 5]}$. 高 亮度和生物相容性的无机和有机近红外苂光材料的设 计和开发成为驱动近红外生物成像发展的原动力. 目
前，近红外生物成像探针包括有机小分子苂光探

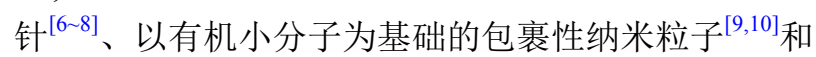

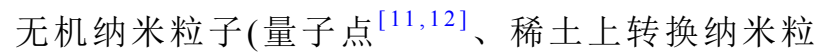

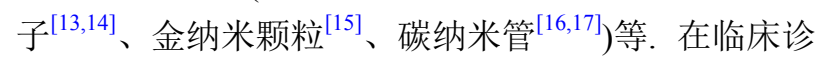
断和外科手术苂光导航中 ${ }^{[18,19]}$, 这些探针已经展示出 近红外生物成像的优势, 有望替代目前广泛应用的在 可见光区域发光的生物成像探针. 然而, 近红外有机 小分子通常都存在光漂白、斯托克斯(Stokes)位移 小、菼光寿命较短、光稳定性较差等问题; 无机纳米 材料由于其自身极小尺寸带来的代谢毒性等. 因此,

引用格式: Zhu XF, Jin GQ, Zhang JL. Design and applications of NIR lanthanide molecular probes for bioimaging and biosensing. Sci Sin Chim, 2020, 50: 15751584, doi: 10.1360/SSC-2020-0118 
发展具有高效近红外吸收和发射的探针，特别是具有 波长超过 $1000 \mathrm{~nm}$ 苂光信号的探针，是近红外生物成 像发展的关键. 发展同时拥有有机小分子和金属纳米 粒子二者优点的近红外金属(稀土)配合物生物荧光探 针, 将极大地丰富近红外荧光探针的种类, 推动近红外 生物苂光成像的快速发展.

稀土元素具有独特的 $4 \mathrm{f}$ 亚层电子结构, 是光、 电、磁领域的“明星”元素，在能量转换、传输、存储 等方面具有广泛的用途. 通过选择和设计合适的配体, 对稀土离子配位场进行调节，可在分子层面上调控稀 土的物理性质. 以发光为例，稀土发光配合物具有大 Stokes位移、长荧光寿命、窄发射谱带、耐光漂白等 光物理性质，在苂光传感、生物成像等领域得到了广 泛的研究和应用. 尤其是在可见光区域, $\mathrm{Eu}^{3+}$ 和 $\mathrm{Tb}^{3+}$ 发 光配合物的量子产率较高，已经被设计成 $\mathrm{pH}$ 响应探 针 $^{[20,21]}$ 、金属离子探针 ${ }^{[22,23]}$ 、阴离子探针 ${ }^{[24,25]}$ 、有机 小分子探针 ${ }^{[26]}$ 等. 一些功能性稀土荧光探针已经被应 用于生物医学 ${ }^{[27]}$ 和生物成像领域 ${ }^{[28]}$. 此外, 也有少量 关于可见光稀土配合物在活体成像中应用的报道 ${ }^{[29]}$.

受制于配体敏化效率不高、近红外发射易于被高 能 $\mathrm{C}-\mathrm{H}(\mathrm{X}=\mathrm{C}, \mathrm{O}, \mathrm{N})$ 键振动淬灭等问题, 相对而言, 近 红外区域的发光稀土配合物的研究一直滞后于可见区 域发光的稀土配合物. 近年来, 近红外发光稀土配合物 的生物应用逐步发展起来，并逐渐扩展应用到NIR生 物成像、生物传感 ${ }^{\left[{ }^{[0]}\right.}$ 及肿瘤、血管、淋巴结活体成 像等方面 ${ }^{[31]}$. 例如，Wong 等 ${ }^{[32]}$ 和Maury等 ${ }^{[33,34]}$ 先后合 成了一系列 $\mathrm{Yb}$ 配合物并应用于活细胞内的单/双光子 成像. 自2013年开始, Petoud和Pecoraro课题组 ${ }^{[35 \sim 38]}$ 在 稀土配合物的近红外生物成像应用方面做了大量研究 工作，报道了以冠醚和氮杂冠醚为配体合成的分子簇 活细胞成像探针的应用 ${ }^{[38]}$. 另外，香港大学支志明课 题组 ${ }^{[39]}$ 也报道了镱一八乙基吓啉配合物可作为诱导细 胞凋亡的试剂，打开了镱的卟啉化合物作为金属药物 的大门. 本课题组 ${ }^{[1 \sim 3]}$ 则开展了以吓啉类及其衍生物为 配体的稀土荧光探针的设计、合成，以及在活体水平 高分辨率荧光强度成像和时间分辨荧光寿命成像中的 应用. 尽管稀土配合物在生物医学中的应用逐渐受到 重视, 但是相比于稀土发光纳米材料, 稀土分子探针研 究目前仍处于起步阶段, 存在诸多挑战. 主要包括: (1) 稀土配合物在体内的药代动力学尚不清楚; (2) 高亮度 近红外稀土成像探针的合成困难; (3) 适用于生物体微
环境下检测如 $\mathrm{pH}$ 值、金属离子、活性氧物种和生物分 子等测试的探针分子设计等 ${ }^{[00-42]}$. 在此, 我们结合本课 题组的工作, 综述了近 5 年来近红外稀土荧光探针的设 计和在医学生物成像和生物传感方面的应用.

\section{2 稀土的发光特点}

稀土包含 17 种金属元素, 包括从镧 $(\mathrm{La}) \rightarrow$ 镥 $(\mathrm{Lu})$ 的 15 个镧系元素及与镧系元素化学性质相近的钎 (Y)和 钪(Sc)(图1). 稀土元素的原子最外层 $6 \mathrm{~s}^{2}$ 电子轨道和次 外层 $5 \mathrm{~s}^{2} 5 \mathrm{p}^{6}$ 电子轨道全充满, $5 \mathrm{~d}$ 层全空或者只有一个 电子, 即除镧 $(\mathrm{La}) 、$ 铈 $(\mathrm{Ce}) 、$ 钝 $(\mathrm{Gd}) 、$ 镥 $(\mathrm{Lu}) 4$ 个元素 在 $5 \mathrm{~d}$ 轨道上各有一个电子外, 其他稀土元素的 $5 \mathrm{~d}$ 轨道 上没有电子, 内层 $4 \mathrm{f}$ 轨道上的电子数从 $0 \rightarrow 14$. 稀土元 素拥有未充满的受到外层屏蔽的 $4 \mathrm{f} 、 5 \mathrm{~d}$ 电子轨道, 因 此有丰富的电子能级和长寿命激发态. 稀土离子光吸 收和发射主要来源 $4 \mathrm{f}^{n}$ 组态内的 $\mathrm{f}-\mathrm{f}$ 跃迁、 $4 \mathrm{f}^{n}$ 组态与 $4 \mathrm{f}^{n-1} 5 \mathrm{~d}$ 组态间的 $5 \mathrm{~d}-4 \mathrm{f}$ 跃迁，其吸收或发射可以覆盖紫 外光、可见光到近红外区各种波长(特别是在可见光 区有很强的发射能力), 因而稀土发光材料呈现丰富多 变的光学特性, 但4f-4f跃迁在选律上是禁阻的, 因此稀 土离子发光具有消光系数小、谱线强度较弱(发光效 率不高)、发射峰窄而尖以及跃迁概率很小、苂光寿 命较长等特点 ${ }^{[43]}$.

\section{3 稀土配合物的敏化过程}

由于稀土离子的f-f跃迁是禁阻的，导致其消光系 数很小、发光亮度弱, 极大地限制了稀土离子在生物

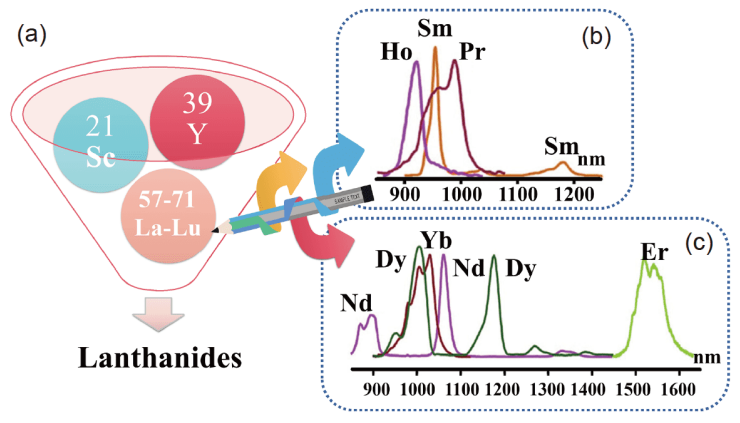

图 1 (a) 17种稀土元素及 $(b, c)$ 一些三价稀土离子在近红外 区的特征发光光谱(网络版彩图)

Figure 1 (a) 17 lanthanide elements and (b, c) the emissions of some trivalent lanthanide ions in NIR region (color online). 
领域方面的应用 ${ }^{[44,45]}$. Weissman ${ }^{[46]}$ 在1942年提出了“天 线效应”的概念, 利用消光系数较大且能级可调的有机 配体与稀土离子配位，通过配体到稀土激发态的能量 传递，提高稀土离子的发光强度. Crosby和Whan等 ${ }^{[47]}$ 对稀土配合物发光进行大量研究后，将能量传递过程 归纳为“配体单线态 $\rightarrow$ 配体三线态 $\rightarrow$ 稀土激发态”能量 传递路径. 如图2所示, 配体受光激发吸收辐射能, 从单 重态的基态 $\mathrm{S}_{0}$ 跃迁至激发态 $\mathrm{S}_{n}$ 的一个振动能级, 经系 间窝越(ISC) 到达三线态激发态 $\left(\mathrm{T}_{n}\right) . \mathrm{T}_{n}$ 以内转换的方 式弛豫到最低激发三重态 $\mathrm{T}_{1}$, 旋即将能量传递给稀土 离子的激发态. 最后处于激发态的稀土离子以发光的 方式回到基态时，便发射各离子的特征苂光(图2) ${ }^{[48]}$. 由此可见，实现稀土配合物中稀土离子高效特征发光 的必要条件是: 配体最低的 $\mathrm{T}_{1}$ 激发态的能级必须高于 稀土离子的 $\mathrm{f}$ 轨道的 $\mathrm{Ln}^{*}$ 激发态能级. 稀土配合物总的 发光效率 $\Phi_{\mathrm{tot}}$ 主要受到稀土非辐射跃迁的控制, 在数值 上等于配体的敏化效率 $\left(\eta_{\mathrm{sen}}\right)$ 与稀土固有发光量子产率 $\left(\Phi_{\mathrm{Ln}}\right)$ 的乘积(式(1)). 由于整个能量传递过程存在多种 辐射跃迁和非辐射弛豫竞争, 因此设计高效的稀土发 光配合物需要考虑三方面的内容: (1) 选择具有尽可 能大的摩尔消光系数的天线分子; (2) 增大 $\eta_{\mathrm{sen}}$; (3) 减 少非辐射过程, 如低能配体到金属的电荷转移(LMCT) 和 $\mathrm{C}-\mathrm{O} / \mathrm{O}-\mathrm{H} / \mathrm{N}-\mathrm{H}$ 振动, 增大 $\Phi_{\mathrm{Ln}}$.

$\Phi_{\text {tot }}=\eta_{\text {sen }} \times \Phi_{\text {Ln }}$

其中, $\Phi_{\mathrm{tot}}$ 是配合物总的发光效率; $\eta_{\mathrm{sen}}$ 是配体的敏化效 率; $\Phi_{\mathrm{Ln}}$ 是稀土固有发光量子产率. 其中, 敏化效率 $\eta_{\mathrm{sen}}=\Phi_{\mathrm{ISC}} \times \Phi_{\mathrm{ET}}, \Phi_{\mathrm{ISC}}$ 表示系间鼌越效率， $\Phi_{\mathrm{ET}}$ 表示配体 三线态到稀土激发态的传能效率 $\left({ }^{3} \mathrm{~T}^{*} \rightarrow \mathrm{Ln}^{*}\right)$. 由于稀土 的重原子效应, 稀土配合物的系间窝越效率很大, 通常

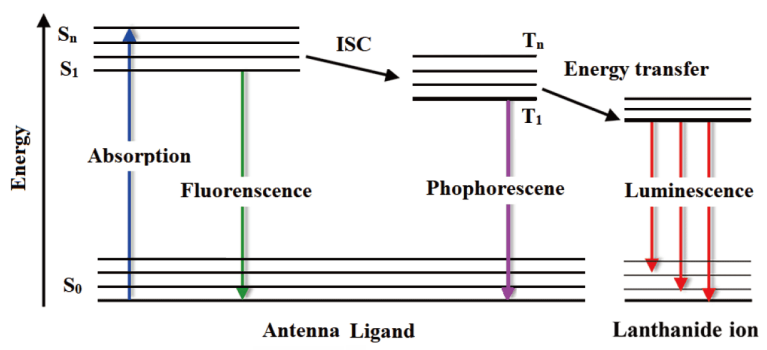

图 2 配体的“天线效应”对稀土金属敏化效应的原理示意 图(网络版彩图)

Figure 2 Simplified Jablonski diagram for the antenna of ligand effect on lanthanide sensitization (color online).
接近于1. 因此提高敏化效率主要可以通过调控配体三 线态与稀土激发态之间的能级差 $\left(\Delta E,{ }^{3} \mathrm{~T}^{*} \rightarrow \mathrm{Ln}^{*}\right)$. 只有 天线配体的三线态能级高于稀土共振能级或与之接近 时, 能量传递才可以发生, 实现敏化. 根据能级差定律, 只有配体三线态能级与稀土激发态的传能效率随能级 差降低而增大 ${ }^{[1,4]}$. 然而, 能级差小于 $1850 \mathrm{~cm}^{-1}$, 则会发 生能量回传, 使传能效率反而降低. 当配体不能有效地 向稀土传能时, 由于稀土的重原子效应, 通常可观察到 配体磷光的发射.

\section{4 卟啉类天线配体的选择与设计}

近红外稀土 $(\mathrm{Ln}=\mathrm{Yb}, \mathrm{Er}, \mathrm{Nd})$ 荧光探针的发光效率 在很大程度上取决于天线配体的电子结构和光物理性 质. 寻找合适的天线配体, 既具有较低 $\mathrm{T}_{1}$ 激发态同时又 可以有效敏化Ln发射, 具有重要意义. 其中, 卟啉配体 具有吸光系数大、配位能力好、三线态能级可调的优 点, 被看作是理想的敏化近红外稀土的天线配体. 由于 卟啉的吸收处于可见区域, 因此, 本课题组一直致力于 仿叶绿素的卟吩内酯衍生物的性质研究. 研究发现, 与 卟啉化合物相比, 引入内酯基团可以显著提高 $\mathrm{Yb}^{3+}$ 的 近红外发射 $(50 \%$ 120\%), 并可以通过有效降低配体的 三线态能级, 提高了 $\mathrm{Yb}^{3+}$ 的敏化效率 ${ }^{[49]}$. 这一工作证明 了内酯基团的引入可以增强 $\operatorname{Ln}$ 的发光量子产率，同时 也为进一步开发应用于体内检测的可逆生物近红外探 针提供了启示. 我们发现葡萄糖氧化酶(GOx) 能激活 该镱生物探针的近红外发射，然后通过填加葡萄糖被 淬灭, 在水相中实现近红外区域对葡萄糖的检测. 因 此，我们发现，通过对卟吩内酯分子对称性和区域异 构效应的调控，可以精准地调控配体激发态的性质， 使之有效敏化近红外稀土发光 ${ }^{[1 \sim 3,50 \sim 52]}$.

\section{1 卟吩内酯的区域异构效应}

卟吩内酯基团的不同取向会造成卟啉吸收光谱的 改变 ${ }^{[53]}$. 通过进一步对Pd和Pt的卟吩二内酯金属配合 物的光谱研究, 证明了内酯基团相对位置(即区域异 构)会影响配体三线态性质 ${ }^{[54]}$. 从卟吩内酯 $\rightarrow$ cis-卟吩 二内酯 $\rightarrow$ trans-卟吩二内酯，三线态逐渐降低，磷光发 射峰红移, 三线态到基态的能级差减小.

为进一步研究区域异构效应对近红外稀土传能效 率的影响，我们设计合成了 cis/trans-Ln-卟吩内酯化合 
物 ${ }^{[5]}$. Y $\mathrm{b}$ 化合物中 $\beta$-取代基取向的位置变化对卟啉的 $\mathrm{T}_{1}$ 和 $\mathrm{T}_{1} \rightarrow \mathrm{Yb}^{*}$ 能量转移过程有显著的影响, cis $-\mathrm{Yb}$ 的 $\mathrm{T}_{1} \rightarrow \mathrm{Yb}^{*}$ 的能隙(约 $1152 \mathrm{~cm}^{-1}$ ) 远大于trans $-\mathrm{Yb}$ (约 $-25 \mathrm{~cm}^{-1}$ ). cis/trans-Ln-卟吩内酯可以“开/关”(cis/ trans) $\mathrm{Yb}^{3+}$ 近红外发射，传能效率约为 $84 \%$ 和 $12 \%$, 量 子产率 $(\mathrm{QY})$ 分别为 $3.3 \%$ 和 $0.4 \%$. 这些结果证明，可以 通过调节内酯基团的区域异构效应精准地调控卟啉的 三线态性质, 改变卟啉的 $\mathrm{T}_{1}$ 态与 $\mathrm{Ln}^{*}$ 发射能级之间的能 级差 $\Delta E\left(\mathrm{~T}_{1}-\mathrm{Ln}^{*}\right)$ 及传能效率(图3). 这为优化和设计近 红外发光稀土探针提供了新思路.

\section{2 高能振动键对稀土发光的影响}

配体和溶剂中距离稀土中心较近的高能振动化学 键 $\mathrm{X}-\mathrm{H}(\mathrm{X}=\mathrm{C}, \mathrm{N}, \mathrm{O})$, 能有效地淬灭稀土离子尤其是近 红外稀土离子的发光, 导致本征量子产率较低 ${ }^{[56,57]}$ (图 4). 为了去除或减少稀土离子附近高能的 $\mathrm{X}-\mathrm{H}$ 振动, 可 以选择重原子(卤代或氝代)取代的配体. Charbonnière、Seitz等 ${ }^{[88 \sim 60]}$ 一系列研究表明, (1) 减小 $\mathrm{X}-\mathrm{H}$ $(\mathrm{X}=\mathrm{C}, \mathrm{N}, \mathrm{O})$ 键的多声子振动弛豫可以延长稀土元素 的荧光寿命; (2) 通过精细剪裁天线配体可以优化能 量转移过程. 我们研究了一系列以全氟代卟啉为光敏 剂, 部分氛化的Kläui三脚架配体为轴向配体的 $\mathrm{Yb}^{3+}$ “三明治型”化合物 ${ }^{[61]}$. 在 $\mathrm{CD}_{2} \mathrm{Cl}_{2}$ 中，总量子产率可达 $63 \%$, 寿命延长到 $714 \mu \mathrm{s}$. 对配合物的光物理性质和结 构进行了系统的分析表明全氟代卟啉是一种有效的天 线配体, 对 $\mathrm{Yb}^{3+}$ 的敏化效率约高达 $100 \%$. $\beta$-吡咯 $\mathrm{C}-\mathrm{F}$ 键 取代C-H键在抑制振动淬灭提高 $\mathrm{Yb}^{3+}$ 近红外发射量子 产率中起到关键作用.

我们合成了 $\beta$-全氟代的吓吩内酯为天线分子的 $\mathrm{Yb}$ 化合物 ${ }^{[62]}$, 在 $\mathrm{CD}_{2} \mathrm{Cl}_{2}$ 中, 总量子产率可达 $58 \%$, 寿命 延长到525 $\mu \mathrm{s}$. 在此基础上利用DIABL-H进行还原, 得 到了两种由于 $\beta-\mathrm{OH}$ 取向不同的立体异构体配合物 Yb$\mathbf{u p} / \mathbf{d o w n} \mathbf{n}^{[63]}$. 光谱分析发现， $\beta-\mathrm{OH}$ 取代基朝向的不同 既不会改变两者的吸收光谱, 也不会影响它们的 $\mathrm{T}_{1}$ 态 能级. $\beta-\mathrm{OH}$ 的存在对于稀土中心的发光有强淬灭作 用. 由于Yb-up分子内氢键的存在会缩短 $\beta-\mathrm{OH}$ 与 $\mathrm{Yb}^{3+}$ 中心的距离，促进了中心离子激发态的非辐射失活. 因此Yb-down的量子产率和荧光寿命 $(4.5 \%, 41 \mu \mathrm{s})$ 是 Yb-up的两倍 $(2.4 \%, 20 \mu \mathrm{s})$. 有趣的是, 由于 $\mathrm{O}-\mathrm{H}$ 键的 振动对温度和黏度的高灵敏度, 降低温度和提高溶剂 的黏度均可以抑制O-H基团的振动. Yb-up/down异构
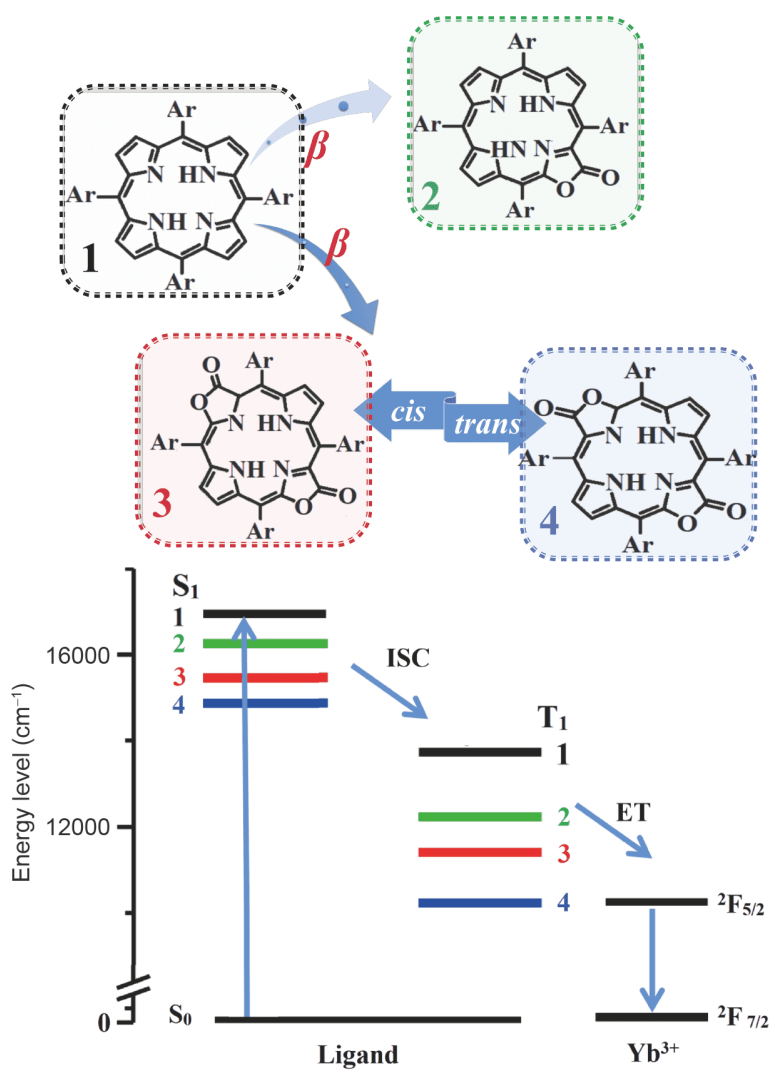

图 3 改变 $\beta$-取代基的个数和取向调控卟啉的 $\mathrm{T}_{1}$ 态的示意 图(网络版彩图)

Figure 3 Schematic diagram of adjusting the porphyrinic $T_{1}$ energy state of porphyrin by changing the number and orientation alteration of $\beta$-substituent (color online).

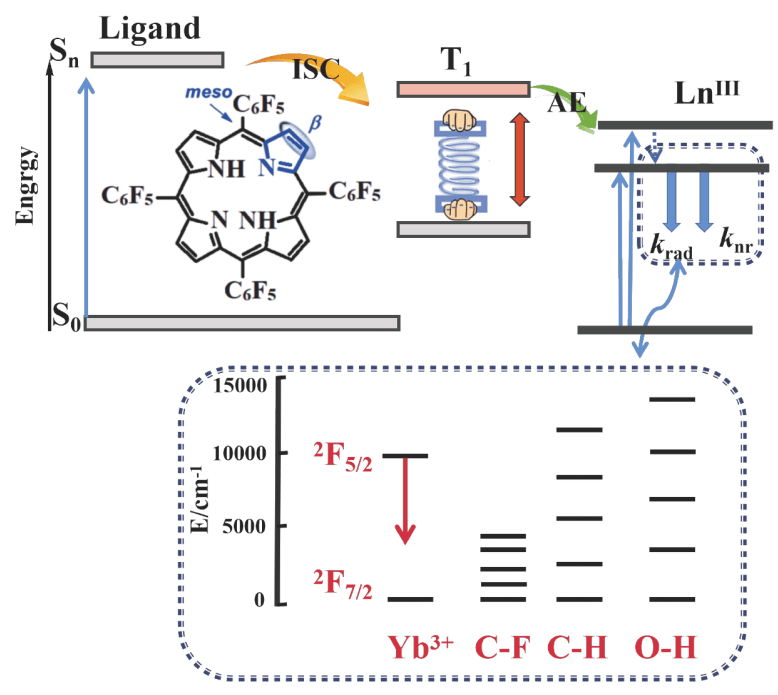

图 4 化学键振动能量对稀土非辐射跃迁过程的影响(网络 版彩图)

Figure 4 Effect of high energy vibration oscillators on non-radiative transition process of rare earth (color online). 
体被发现有潜力作为对外界或环境刺激敏感的NIR$\mathrm{Yb}$ 光学温度计和黏度传感器. 然而, 由于氟化后的溶 解度和细胞摄取率较低, 这些 $\mathrm{Yb}^{3+}$ 配合物在生物成像 中的应用仍然具有挑战性(图5).

\section{5 近红外稀土分子探针在生物成像方面的 应用}

由于Ln-化合物具有独特的光学性质, 尖锐的发射 带、激发波长和发射波长之间的巨大差异、较长的衰 变寿命以及对光漂白的强大抵抗力，使得稀土配合物 活体成像和检测方面具有许多优势，可广泛用于生物 成像和生物传感。本部分简单总结了稀土化合物在 NIR区域的光学成像，讨论了这些探针的发光性能、 毒性以及生物学方面的应用.

\section{1 细胞及活体苂光强度成像}

NIR发射稀土元素(Ln)配合物结合了有机小分子 和无机金属离子的优点，可以实现在近红外区域荧光 成像. 尽管通过改变配体的构型、配位数调整、分子

(a)
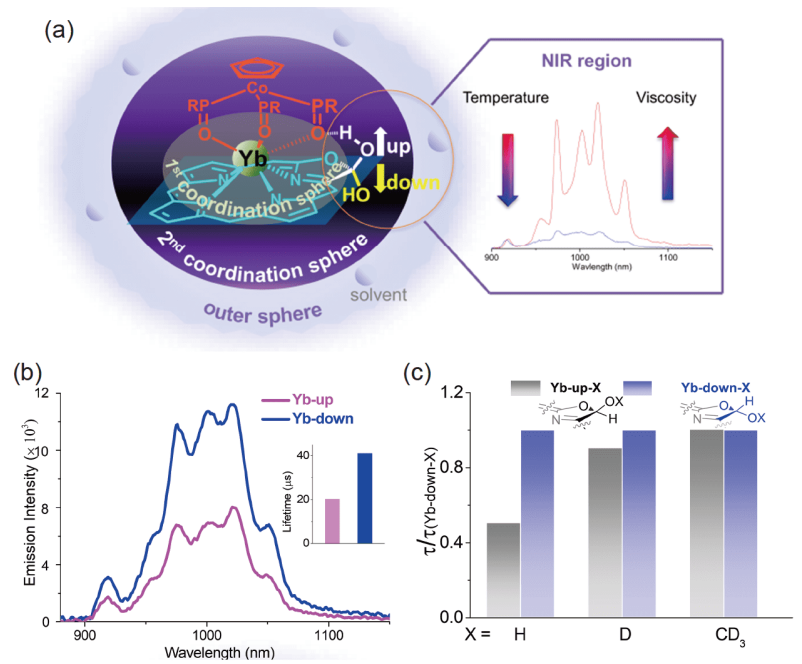

图 5 (a) Yb-up/down的结构式; (b) 化合物Yb-up/down在 二氯甲烷中的发射光谱; (c) 化合物 Yb-up/down、Yb-up/ down-D和Yb-up/down-CD 在二氯甲烷中的衰减寿命的差 值(网络版彩图)

Figure 5 (a) The structures of Yb-up and Yb-down. (b) Luminescence intensity and decay lifetime monitored at $974 \mathrm{~nm}$ of Yb-up and Yb-down in $\mathrm{CH}_{2} \mathrm{Cl}_{2}$ at room temperature $\left(\lambda_{\mathrm{ex}}=406 \mathrm{~nm}, A_{406 \mathrm{~nm}}=0.1\right)$. (c) Decay lifetime of Yb-up-X referred to Yb-down-X $\left(\mathrm{X}=\mathrm{H}, \mathrm{D}, \mathrm{CD}_{3}\right)$ monitored at $974 \mathrm{~nm}$ in $\mathrm{CH}_{2} \mathrm{Cl}_{2}$ at room temperature (color online).
内能量转移、分子内光诱导电子转移等策略，可以增 加稀土配合物的发光强度和荧光寿命. 由于Ln化合物 的量子产率低和成像探测器的限制，近红外稀土化合 物在活体成像中的应用才刚刚起步，但细胞成像实现 了从体外设计到体内成像的过渡。2014年，Eliseeva 等 ${ }^{[64]}$ 报道的多核树状的 $\mathrm{Sm}^{3+}$ 纳米颗粒，可同时应用于 可见和近红外细胞成像. Petoud等 ${ }^{[65,66]}$ 将 $\mathrm{Yb}^{3+}$ 发色团 包裹在聚苯乙烯微球中. 修饰过的含有天线配体和稀 土离子的纳米粒子或团簇能够进入活细胞，并在原位 获得稀土敏化配体. Wong 等 ${ }^{[32]}$ 发现一种 $\mathrm{Yb}^{3+}$ 配合物与 罗丹明B相结合可用于近红外可见双光子活体细胞成 像. Maury等 ${ }^{[33]}$ 制备的水溶性 $\mathrm{Yb}^{3+}$ 配合物可用于双光 子细胞成像及近红外生物成像. 通过前面的研究发现, 卟啉上的meso取代基的 $\mathrm{C}-\mathrm{H}$ 键对稀土元素的发光影响 较小, 而卟啉中的 $\beta$-吡咯 $\mathrm{C}-\mathrm{H}$ 键和Kläui配体的亚磷酸 $\mathrm{C}-\mathrm{H}$ 键对 $\mathrm{Ln}$ 具有显著的淬灭作用. 为了解决这些问题, 分别通过对全氟代镱吓啉的meso-和 $\beta$-位置的修饰, 开 发了一系列具有生物相容性 $\beta$-氟化 $\mathrm{Yb}^{3+}$ 卟啉配合 物 ${ }^{[67]}$. 这些 $\mathrm{Yb}^{3+}$ 配合物显示出高的近红外发光(在 $\mathrm{DMSO}$ 中 $\Phi_{\mathrm{tot}}=23 \%$ ，在 $\mathrm{H}_{2} \mathrm{O}$ 中 $\left.\Phi_{\mathrm{tot}}=13 \%\right)$ 和较高的荧光 寿命 $(249 \mu \mathrm{s})$, 同时应用于荧光强度成像和荧光寿命成 像. 为了提高近红外稀土配合物的量子产率, 我们需要 进一步研究开发具有良好量子产率和生物相容性的近 红外发光Ln探针. 尽管本课题组 ${ }^{[61,67]}$ 开发了高亮度的 全氟夹心卟啉镱配合物，但全氟取代使其疏水性太强， 无法被细胞吸收. 因此, 我们通过三种合成策略, 成功 地合成了一系列生物相容性好、高量子产率的镱卟啉 化合物(图6) ${ }^{[67]}$. 在细胞实验方面, 我们注意到附加氨 基的 $\mathrm{Yb}$ 化合物对 $\mathrm{HeLa}$ 细胞的 $\mathrm{IC}_{50}$ 值低至 $1.0 \mu \mathrm{M}$, 这是 由单线态氧产生的光毒性引起的，而其他化合物即使 在 $10 \mu \mathrm{M}$ 浓度(细胞活力 $>80 \%$ )的光辐射下也表现出可 忽略的细胞毒性. 这系列配合物在近红外活细胞成像 中展示出高亮度和长寿命的特点, 显示出比非氟代 $\mathrm{Yb}^{3+}$ 配合物更高的近红外细胞内发光.

$\mathrm{Hao}$ 等 ${ }^{[68}$ 报道了 Nd-DTPA化合物的体内行为，该 化合物显示出快速的肾脏排泄和肿瘤积聚特性. 同年 Zhang等 ${ }^{[69]}$ 发现了类似的结构也显示出快速的肾排泄. 然而, 由于这两种体系中缺少天线配体, 使得Stokes位 移小、吸收系数低、对激发激光功率要求较高.

我们将水溶性 $\beta$-氟化 $\mathrm{Yb}^{3+}$ 卟啉进一步应用于小动 物全身苂光成像 ${ }^{[70]} . \mathrm{Yb}$-化合物在水中的量子产率约 

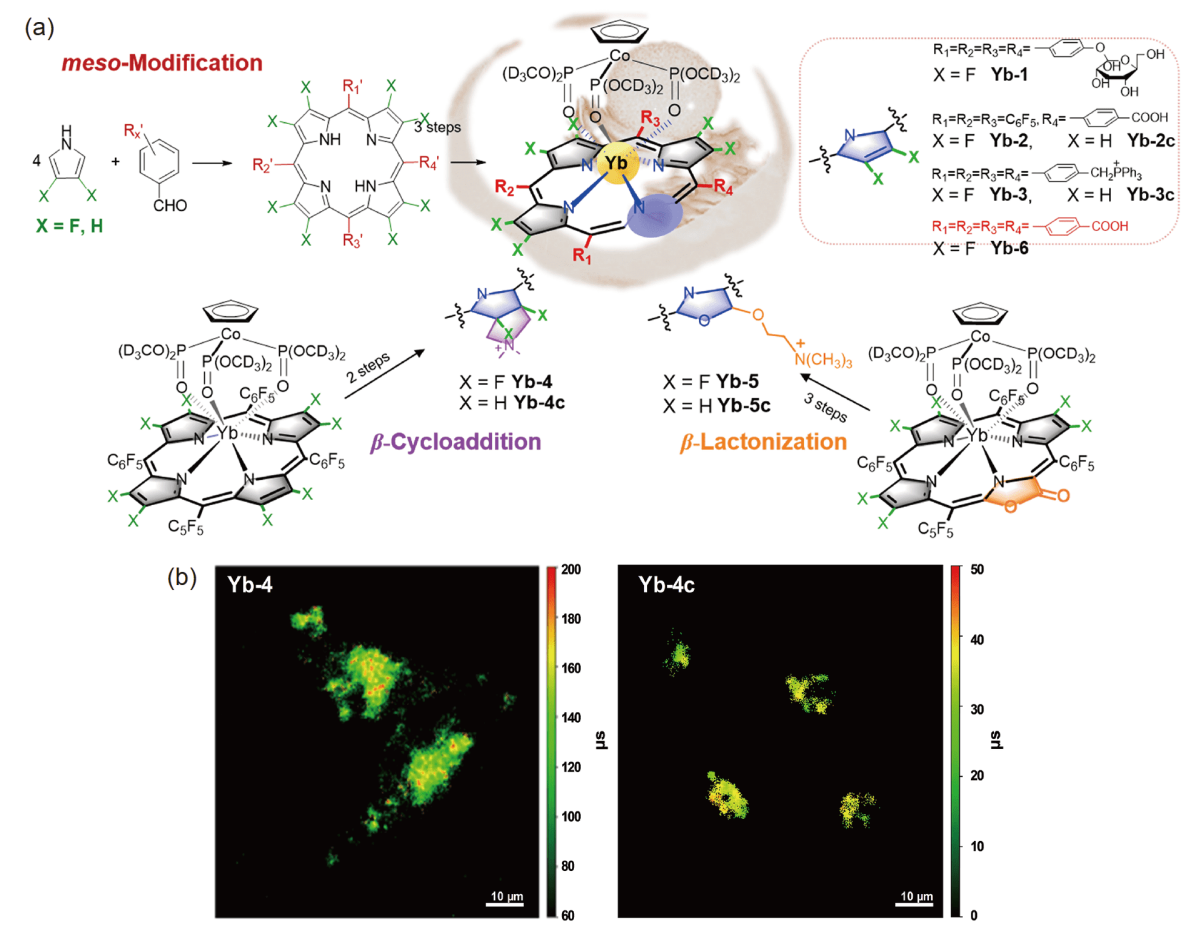

图 6 (a) 具有生物相容的 $\beta$-氟代 $Y b^{3+}$ 配合物Yb-1-5和相应的 $\beta$-氢代Y $\mathrm{b}^{3+}$ 配合物 $Y \mathbf{b}-2 \mathrm{c}-5 \mathbf{c}$ 的结构式及合成路线示意图. (b) 用 $10 \mu \mathrm{M}$ Yb-4和Yb-4c孵育HeLa细胞的时间分辨近红外荧光寿命成像 $\left(\lambda_{\mathrm{ex}}: 408 \mathrm{~nm} ; \lambda_{\mathrm{em}}\right.$ : $935 / 170 \mathrm{~nm}$ 带通; 曝光时间: $4 \mathrm{~ms}$ ). 标尺: $10 \mu \mathrm{m}^{[67]}$ (网络版彩图)

Figure 6 (a) The structures and the synthetic routes for biocompatible $\beta$-fluorinated $\mathrm{Yb}^{3+}$ complexes $\mathbf{Y b}$-1-5 and $\beta$-hydrogenated analogues $\mathbf{Y b}$-2c5c. (b) NIR time-resolved images of living HeLa cells incubated with $10 \mathrm{mM}$ Yb-4 and $\mathbf{Y b}-\mathbf{4 c}\left(\lambda_{\mathrm{ex}}: 408 \mathrm{~nm} ; \lambda_{\mathrm{em}}\right.$ : $935 / 170 \mathrm{~nm}$ bandpass; dwell time: $4 \mathrm{~ms}$ ). Scale bar: $10 \mathrm{~mm}$ [67] (color online).

为 $10 \%$, 在 $\mathrm{Q}$ 带激发时显示出 $>3 \mathrm{~mm}$ 的穿透深度. 这种 化合物就像大多数有机苂光团一样，可以通过肝胆和 肾脏系统代谢. 值得一提的是, 我们将Yb化合物应用 于荧光引导了原位淋巴组织手术，这是第一个用于全 身生物成像和手术导航的NIR-II稀土元素分子探针 (图7).

\section{2 时间分辨菼光寿命成像}

苂光寿命是荧光物质的固有性质，不受到化合物 发光强度、光漂白、激光光强、检测器狭缝等因素的 影响, 能够定量显示生理环境的波动变化 ${ }^{[71,72]}$. 苂光寿 命成像具有很高的时空分辨率, 可以实现定量测量. 因 此，时间分辨苂光寿命成像(FLIM)已广泛应用于稳态 苂光成像难以观察的细胞事件的可视化. Ln离子通常 比生物自荧光具有更长的寿命 $\left(10^{-6} \sim 10^{-2} \mathrm{~s}\right)$, 特别适合 苂光寿命成像. 截至目前, 关于近红外活体内苂光标记 的报道很少. 2015 年, Sørensen等 ${ }^{[73]}$ 将钕和镱配合物负
载到硅纳米颗粒中, 实现了纳米颗粒的近红外荧光寿 命成像. 2017年, Maury等 ${ }^{[74]}$ 报道了一种 $\mathrm{Tb}^{3+}$ 化合物, 通过分子旋转检测细胞内黏度, 但后续实验证明实际 上是溶剂极性导致的 $\mathrm{Tb}$ 发光寿命改变.

2019年，本课题组 ${ }^{[75]}$ 通过将羧酸基团引入卟啉的 $m e s o$-位置, 设计合成了水溶性的 $\beta$-氟代 $\mathrm{Yb}$ 卟啉化合 物, 并研究了该化合物对 $\mathrm{pH}$ 的响应性质. 当 $\mathrm{pH}$ 值从 9.0 到 5.0, 化合物表现出对 $\mathrm{pH}$ 的敏感响应, 增加了卟啉的 近红外发射和寿命 $(13.4 \sim 170 \mu \mathrm{s})$. 当继续减小 $\mathrm{pH}$ 值, 虽 然也增加了卟啉的寿命 $(135 \sim 170 \mu \mathrm{s})$, 但可能由于在酸 性水溶液中该化合物降低了溶解度而形成了聚集体, 因此发生了NIR淬灭(图8).

本课题组 ${ }^{[75]}$ 将这种宽范围 $\mathrm{pH}$ 敏感的 $\mathrm{Yb}^{3+}$ 分子寿命 探针用于体内胃肠道 $\mathrm{pH}$ 检测. 用近红外荧光探针研究 了抗酸药水铝碳酸镁片对胃肠道 $\mathrm{pH}$ 值的调节作用. 与 投加铝碳酸镁片后荧光强度成像的模糊结果相比, 根 据胃内 $\mathrm{pH}$ 值从1.5到6.5到1.5的变化, $\mathrm{Yb}$ 在胃内的荧光 

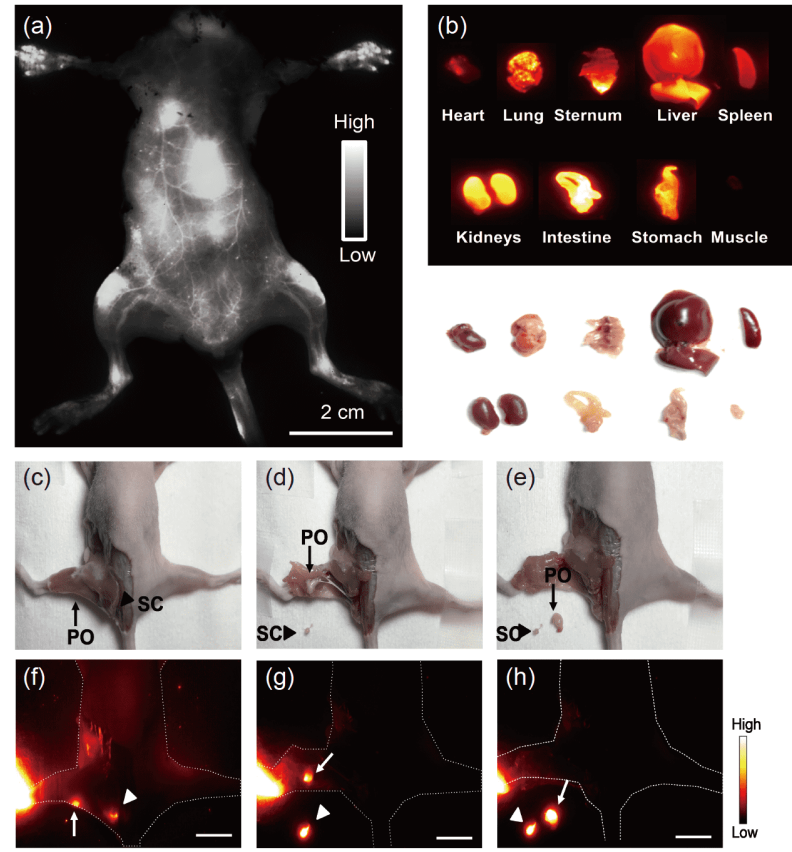

图 $7 \beta$-氟化 $\mathrm{Yb}^{3+}$ 化合物已成功用于体内成像, 并在荧光成 像指导下完成了淋巴结手术 ${ }^{[70]}$. (a) Yb-6 $(150 \mu \mathrm{L}, 3 \mathrm{mg} / \mathrm{mL})$ 的经C57BL/6小鼠静脉内注射 $5 \mathrm{~min}$ 后全身NIR-II荧光图像 $\left(\lambda_{\mathrm{ex}}, 520 \mathrm{~nm} ; \lambda_{\mathrm{em}}, 1000 \mathrm{~nm}\right.$ 长通滤光片; $3000 \mathrm{~ms}$ 曝光; (b) 注 射Yb-6后 $12 \mathrm{~h}$ 的离体生物分布研究; 活体NIR-II图像引导的 小鼠腿弯部和骨淋巴结成像和活检. 裸鼠左肢注射 $3 \mathrm{mg} / \mathrm{mL}$ $\mathrm{Yb}-6$ 后约 $24 \mathrm{~h}$ 的NIR-II苂光图像(每组 $n=3 ; \lambda_{\mathrm{ex}}, 520 \mathrm{~nm} ; \lambda_{\mathrm{em}}$, $1000 \mathrm{~nm}$ 长通滤光片; $1000 \mathrm{~ms}$ 曝光). (c e ) 照片描绘了俯卧位 置的裸鼠, 用于通过解剖(c)中的皮肤, 然后解剖肌肉来成像 小鼠腿弯部(黑色箭头)和淋巴结(黑色箭头)并暴露(d)和(e) 中的淋巴结; (f h) 小鼠腿弯部(白色箭头)和骨淋巴结(白色 箭头)在短时间内清晰地被近红外荧光识别并解剖. 比例尺: $1 \mathrm{~cm}$ (网络版彩图)

Figure $7 \quad \beta$-Fluorinated $\mathrm{Yb}^{3+}$ complexes were successfully applied in in vivo imaging and helped fluorescence-guided sentinel lymph node surgery [70]. (a) Whole body NIR-II fluorescence images of Yb-2 (150 $\mu \mathrm{L}, 3 \mathrm{mg} / \mathrm{mL})$ after $5 \mathrm{~min}$ intravenous injection into C $57 \mathrm{BL} / 6$ mice $\left(\lambda_{\mathrm{ex}}\right.$ $520 \mathrm{~nm} ; \lambda_{\mathrm{em}}, 1000 \mathrm{~nm}$ longpass filter; $3000 \mathrm{~ms}$ exposure; color bar ranges from 5000 to $40000, n=3$ per group); (b) ex vivo biodistribution studies at $12 \mathrm{~h}$ after the injection of $\mathrm{Yb}-2$. (c-h) In vivo NIR-II imageguided popliteal and sacral lymph node mapping and biopsy. NIR-II fluorescence image at $\sim 24 \mathrm{~h}$ post-injection of $3 \mathrm{mg} / \mathrm{mL} \mathrm{Yb}-2$ in the left foot of nude mice ( $n=3$ per group; $\lambda_{\mathrm{ex}}, 520 \mathrm{~nm} ; \lambda_{\mathrm{em}}, 1000 \mathrm{~nm}$ longpass filter; $1000 \mathrm{~ms}$ exposure; color bar ranges from 1200 to 50000). (c-e) Photograph depicting a nude mouse at a prone position for imaging popliteal (black arrow) and sacral (black arrowhead) lymph nodes by dissecting the skin in (c), then dissecting the muscles and exposing the lymph nodes in (d, e). (f-h) The popliteal (white arrow) and sacral (white arrowhead) lymph nodes were clearly identified and dissected at their precise position in a short time. Scale bar: $1 \mathrm{~cm}$ (color online).

寿命呈现出从 $170 \mu \mathrm{s}$ 到 $130 \mu \mathrm{s}$ 再到 $170 \mu \mathrm{s}$ 的可逆变化. 因此通过使用FLIM，稀土元素寿命探针能够实时、原 位和定量地监测小鼠模型胃内 $\mathrm{pH}$ 值的变化(图9).

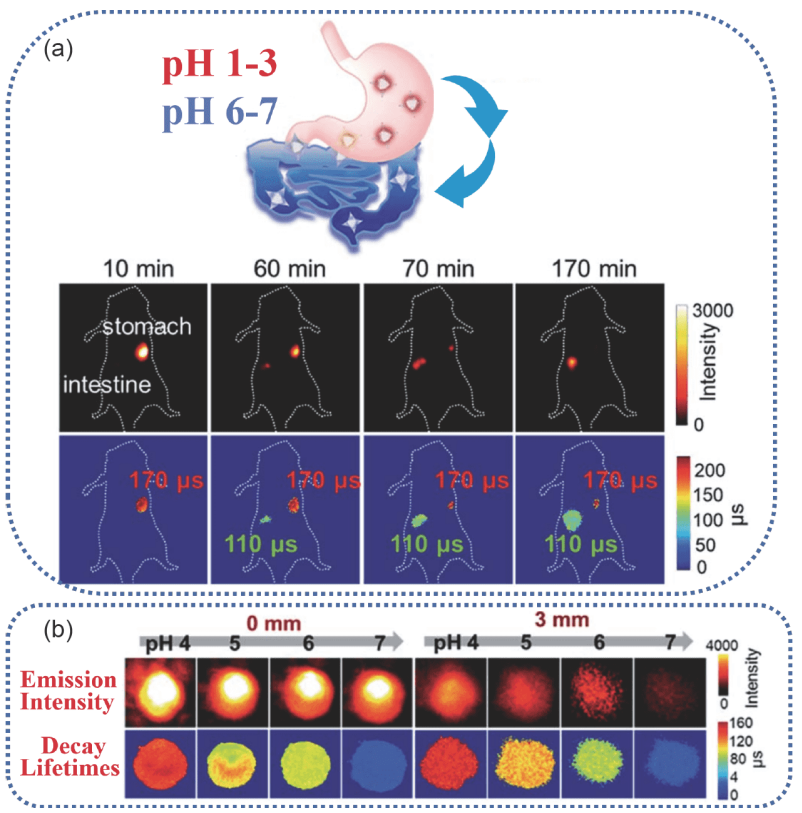

图 8 (a) $\beta$-氟化 $\mathrm{Yb}^{3+}$ 化合物从胃到肠的代谢过程示意图、 近红外荧光强度成像及FLIM图像; (b) 不同 $\mathrm{pH}$ 值和穿透深 度下 $\beta$-氟化 $\mathrm{Yb}^{3+}$ 化合物的发射强度和寿命 ${ }^{[75]}$ (网络版彩图)

Figure 8 (a) Schematic illustration of metabolic process of $\beta$ fluorinated $\mathrm{Yb}^{3+}$ complex from stomach to intestine, NIR fluorescence intensity imaging and FLIM images; (b) emission intensity and lifetime of $\beta$-fluorinated $\mathrm{Yb}^{3+}$ complex in different $\mathrm{pH}$ values and penetration depths [75] (color online).
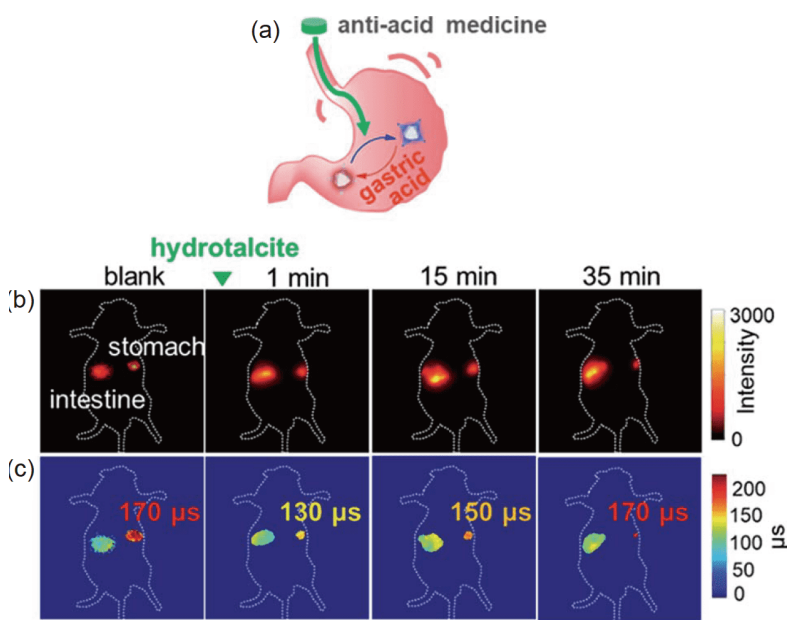

图 9 (a) F-Yb法监测胃酸pH值变化过程示意图. (b) 近红外 荧光成像(曝光时间: $25 \mathrm{~ms}$ ). 顶部的绿色三角形表示喂入的 碳酸铝镁片. (c) FLIM成像(曝光时间: $250 \mathrm{~ms}$ ). $\lambda_{\mathrm{ex}}$ : $532 \mathrm{~nm}$; $\lambda_{\mathrm{em}}: 1000 \mathrm{~nm}$ 长通 $^{[75]}$ (网络版彩图)

Figure 9 (a) Schematic illustration of monitoring pH changes by F$\mathbf{Y b}$ in the anti-acid process in stomach. (b) NIR intensity fluorescence imaging (exposure time, $25 \mathrm{~ms}$ ). The green triangle at the top indicates the gavage of hydrotalcite. (c) FLIM images (exposure time, $250 \mathrm{~ms}$ ). $\lambda_{\mathrm{ex}}: 532 \mathrm{~nm} ; \lambda_{\mathrm{em}}: 1000 \mathrm{~nm}$ longpass [75] (color online) 


\section{3 影像学及医学应用}

传统上, 疾病的检测与治疗互相独立. 究其本源, 前者需要物理信号的反馈，而后者则利用了一定的生 物化学反应. 近年来, 诊疗一体化(theragnostics)逐渐 兴起，向我们展示了如何将医学诊断和治疗合二为一, 以“相辅相成”的方式提高疾病防控的可能性. 然而, 不 同功能的分子往往化学结构相差很大 ${ }^{[76]}$.

我们之前发现在卟啉的 $\beta$ 位引入内酯基团, 精确调 控内酯基团的个数和相对取向, 可以有效改变化合物 的光物理性质，在一定程度上实现了在上转换发光和 稀土敏化发光中“turn on”和“turn off”的切换. 深入研 究显示, 调控卟吩二内酯的外围修饰, 可以进一步放大 这一差异. 我们设计了新的合成路线, 利用 1,3 -偶极环 加成同一个化学反应，得到了两种新型顺式、反式卟 吩二内酯异构体, 并通过近红外光学成像以及光动力 治疗实验，验证了两个异构体在检测与治疗中的功能 裂分, 以及二者不需分离的混合物直接作为检测-治疗 一体试剂进行应用的可能，实现了“同形异用”的设 想 ${ }^{[77]}$ (图10).

\section{6 总结与展望}

本文综述了稀土分子探针在近红外区域生物成像 方面的研究进展. 通过对卟酚内酯meso- $\beta$-位置的区域 异构、氟代等结构优化和调节, $\mathrm{Yb}$ 卟啉化合物展示出 优良的近红外发光、抗光漂白等光物理性质. 通过生 物相容性修饰，这些稀土配合物显示出良好的低毒性

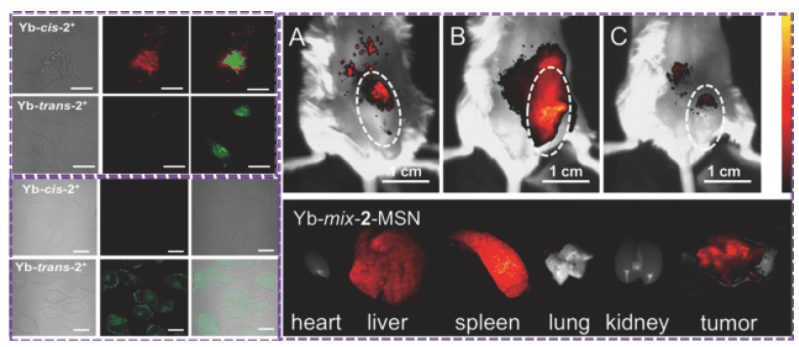

图 10 Yb-cis-/trans-2 ${ }^{+}$化合物在HeLa细胞中的稳态苂光成 像、 $\mathrm{H}_{2} \mathrm{DCFDA}$ 苂光成像以及在注射Yb-cis-/trans-2-MSN后 $48 \mathrm{~h}$ 肿瘤鼠成像 $(\mathrm{A} \sim \mathrm{C}$ )和代表性脏器的(底部) NIR 苂光图 像 ${ }^{[77]}$ (网络版彩图)

Figure 10 Steady-state fluorescence imaging of $\mathbf{Y b}$-cis-/trans-2 ${ }^{+}$in $\mathrm{HeLa}$ cells, $\mathrm{H}_{2} \mathrm{DCFDA}$ fluorescence images of HeLa cells. In vivo (AC) and representative ex vivo biodistribution (bottom) NIR fluorescence images recorded $48 \mathrm{~h}$ after the injection of Yb-cis-/trans-2-MSN [77] (color online).

和靶向性，可应用于细胞乃至活体成像. 然而，除 $\mathrm{Yb}^{3+}$ 外，尽管一些 $\mathrm{Er}^{3+} 、 \mathrm{Nd}^{3+} 、 \mathrm{Sm}^{3+}$ 等近红外发射稀土配 合物有较高的量子产率，但这些稀土元素在近红外区 并没有得到很好的开发. 结合超分子化学或配位化学 的分子设计策略，选择合适的天线配体，促进这些近 红外稀土离子化合物在生物学的进一步发展探索, 实 现其在生物成像和生物传感中以及临床上的应用是值 得研究的. 同时, 尽管一些近红外稀土分子探针已经应 用于体内成像，而稀土配合物在体内的药动学和药效 学尚不清楚. 这些工作展示了近红外稀土分子探针在 生物成像和生物传感方面的巨大潜力，进一步研究其 在体内外的应用对近红外稀土配合物临床应用具有重 要意义.

致谢本工作得到了北京大学高性能计算平台的支持, 特此致谢.

\section{参考文献}

1 Ning Y, Zhu M, Zhang JL. Coord Chem Rev, 2019, 399: 213028

2 Jin GQ, Ning Y, Geng JX, Jiang ZF, Wang Y, Zhang JL. Inorg Chem Front, 2020, 7: 289-299

3 Peng XX, Zhu XF, Zhang JL. J Inorg Biochem, 2020, 209: 111118

4 Bunzli JCG. Chem Rev, 2010, 110: 2729-2755

5 Aletti AB, Gillen DM, Gunnlaugsson T. Coord Chem Rev, 2018, 354: 98-120

6 Qi J, Qiao W, Wang ZY. Chem Record, 2016, 16: 1531-1548

7 Schnermann MJ. Nature, 2017, 551: 176-177

8 Yang Q, Hu Z, Zhu S, Ma R, Ma H, Ma Z, Wan H, Zhu T, Jiang Z, Liu W, Jiao L, Sun H, Liang Y, Dai H. J Am Chem Soc, 2018, 140: 1715-1724

9 Hong G, Zou Y, Antaris AL, Diao S, Wu D, Cheng K, Zhang X, Chen C, Liu B, He Y, Wu JZ, Yuan J, Zhang B, Tao Z, Fukunaga C, Dai H. Nat Commun, 2014, 5: 4206-4215 
10 Liu HY, Wu PJ, Kuo SY, Chen CP, Chang EH, Wu CY, Chan YH. J Am Chem Soc, 2015, 137: 10420-10429

11 Li C, Zhang Y, Wang M, Zhang Y, Chen G, Li L, Wu D, Wang Q. Biomaterials, 2014, 35: 393-400

12 Hu J, Ortgies DH, Aguliar Torres R, Fernández N, Porto L, Martín Rodríguez E, García Solé J, Jaque D, Alfonso F, Rivero F. Adv Funct Mater, 2017, 27: 1703276

13 Dong H, Du SR, Zheng XY, Lyu GM, Sun LD, Li LD, Zhang PZ, Zhang C, Yan CH. Chem Rev, 2015, 115: 10725-10815

14 Dong H, Sun LD, Yan CH. Chem Soc Rev, 2015, 44: 1608-1634

15 Boisselier E, Astruc D. Chem Soc Rev, 2009, 38: 1759-1782

16 Welsher K, Sherlock SP, Dai H. Proc Natl Acad Sci USA, 2011, 108: 8943-8948

17 Gong H, Peng R, Liu Z. Adv Drug Deliver Rev, 2013, 65: 1951-1963

18 Zhu S, Tian R, Antaris AL, Chen X, Dai H. Adv Mater, 2019, 31: 1900321

19 Martinić I, Eliseeva SV, Petoud S. J Lumin, 2017, 189: 19-43

20 Zhou Z, Zhang CC, Zheng Y, Wang Q. Dyes Pigments, 2018, 150: 151-157

21 Gupta K, Patra AK. Eur J Inorg Chem, 2018, 2018: 1882-1890

22 Walter ERH, Williams JAG, Parker D. Chem Eur J, 2018, 24: 7724-7733

23 Aulsebrook ML, Starck M, Grace MR, Graham B, Thordarson P, Pal R, Tuck KL. Inorg Chem, 2019, 58: 495-505

24 Butler SJ, McMahon BK, Pal R, Parker D, Walton JW. Chem Eur J, 2013, 19: 9511-9517

25 Hou Z, Li P, Wang HY, Li Z, Li H. Dyes Pigments, 2017, 147: 429-435

26 Singh K, Goenka A, Ganesh S, Patra AK. Eur J Inorg Chem, 2018, 2018: 3942-3951

27 Eliseeva SV, Bünzli JCG. Chem Soc Rev, 2010, 39: 189-227

28 Amoroso AJ, Pope SJA. Chem Soc Rev, 2015, 44: 4723-4742

29 Surender EM, Comby S, Cavanagh BL, Brennan O, Lee TC, Gunnlaugsson T. Chem, 2016, 1: 438-455

30 Bünzli JCG. Coord Chem Rev, 2015, 293-294: 19-47

31 Alcala MA, Shade CM, Uh H, Kwan SY, Bischof M, Thompson ZP, Gogick KA, Meier AR, Strein TG, Bartlett DL, Modzelewski RA, Lee YJ, Petoud S, Brown CK. Biomaterials, 2011, 32: 9343-9352

Zhang T, Zhu X, Cheng CCW, Kwok WM, Tam HL, Hao J, Kwong DWJ, Wong WK, Wong KL. J Am Chem Soc, 2011, 133: 20120-20122 D’Aléo A, Bourdolle A, Brustlein S, Fauquier T, Grichine A, Duperray A, Baldeck PL, Andraud C, Brasselet S, Maury O. Angew Chem Int Ed, 2012, 51: 6622-6625

Bui AT, Beyler M, Grichine A, Duperray A, Mulatier JC, Guyot Y, Andraud C, Tripier R, Brasselet S, Maury O. Chem Commun, 2017, 53: 60056008

Foucault-Collet A, Shade CM, Nazarenko I, Petoud S, Eliseeva SV. Angew Chem Int Ed, 2014, 53: 2927-2930

Trivedi ER, Eliseeva SV, Jankolovits J, Olmstead MM, Petoud S, Pecoraro VL. J Am Chem Soc, 2014, 136: 1526-1534

Martinić I, Eliseeva SV, Nguyen TN, Pecoraro VL, Petoud S. J Am Chem Soc, 2017, 139: 8388-8391

Martinić I, Eliseeva SV, Nguyen TN, Foucher F, Gosset D, Westall F, Pecoraro VL, Petoud S. Chem Sci, 2017, 8: 6042-6050

Kwong WL, Wai-Yin Sun R, Lok CN, Siu FM, Wong SY, Low KH, Che CM. Chem Sci, 2013, 4: 747-754

Wang X, Chang H, Xie J, Zhao B, Liu B, Xu S, Pei W, Ren N, Huang L, Huang W. Coord Chem Rev, 2014, 273-274: 201-212

Butler SJ, Parker D. Chem Soc Rev, 2013, 42: 1652-1666

Sy M, Nonat A, Hildebrandt N, Charbonnière LJ. Chem Commun, 2016, 52: 5080-5095

Cui Y, Yue Y, Qian G, Chen B. Chem Rev, 2012, 112: 1126-1162

He S, Song J, Qu J, Cheng Z. Chem Soc Rev, 2018, 47: 4258-4278

Rocha J, Carlos LD, Paz FAA, Ananias D. Chem Soc Rev, 2011, 40: 926-940

Weissman SI. J Chem Phys, 1942, 10: 214-217

Crosby GA, Whan RE, Alire RM. J Chem Phys, 1961, 34: 743-748

Moore EG, Samuel APS, Raymond KN. Acc Chem Res, 2009, 42: 542-552

Ke XS, Yang BY, Cheng X, Chan SLF, Zhang JL. Chem Eur J, 2014, 20: 4324-4333

Yang ZS, Ning Y, Yin HY, Zhang JL. Inorg Chem Front, 2018, 5: 2291-2299

Ning Y, Jin GQ, Zhang JL. Acc Chem Res, 2019, 52: 2620-2633

Yao Y, Rao Y, Liu Y, Jiang L, Xiong J, Fan YJ, Shen Z, Sessler JL, Zhang JL. Phys Chem Chem Phys, 2019, 21: 10152-10162

Ke XS, Chang Y, Chen JZ, Tian J, Mack J, Cheng X, Shen Z, Zhang JL. J Am Chem Soc, 2014, 136: 9598-9607

Ke XS, Zhao H, Zou X, Ning Y, Cheng X, Su H, Zhang JL. J Am Chem Soc, 2015, 137: 10745-10752

Ning Y, Ke XS, Hu JY, Liu YW, Ma F, Sun HL, Zhang JL. Inorg Chem, 2017, 56: 1897-1905

Ye HQ, Li Z, Peng Y, Wang CC, Li TY, Zheng YX, Sapelkin A, Adamopoulos G, Hernández I, Wyatt PB, Gillin WP. Nat Mater, 2014, 13: 382- 
386

57 Sveshnikova EB, Ermolaev VL. Opt Spectrosc, 2011, 111: 34-50

Doffek C, Seitz M. Angew Chem Int Ed, 2015, 54: 9719-9721

Souri N, Tian P, Platas-Iglesias C, Wong KL, Nonat A, Charbonnière LJ. J Am Chem Soc, 2017, 139: 1456-1459

Nonat A, Bahamyi

61 Hu JY, Ning Y, Meng YS, Zhang J, Wu ZY, Gao S, Zhang JL. Chem Sci, 2017, 8: 2702-2709

62 Hu JY, Wu ZY, Chai K, Yang ZS, Meng YS, Ning Y, Zhang J, Zhang JL. Inorg Chem Front, 2017, 4: 1539-1545

63 Ning Y, Liu YW, Meng YS, Zhang JL. Inorg Chem, 2018, 57: 1332-1341

64 Foucault-Collet A, Shade CM, Nazarenko I, Petoud S, Eliseeva SV. Angew Chem, 2014, 126: 2971-2974

65 Martinić I, Eliseeva SV, Collet G, Luo TY, Rosi N, Petoud S. ACS Appl Bio Mater, 2019, 2: 1667-1675

66 Foucault-Collet A, Gogick KA, White KA, Villette S, Pallier A, Collet G, Kieda C, Li T, Geib SJ, Rosi NL, Petoud S. Proc Natl Acad Sci USA, 2013, 110: 17199-17204

67 Ning Y, Tang J, Liu YW, Jing J, Sun Y, Zhang JL. Chem Sci, 2018, 9: 3742-3753

68 Li Y, Li X, Xue Z, Jiang M, Zeng S, Hao J. Biomaterials, 2018, 169: 35-44

69 Yang Y, Wang P, Lu L, Fan Y, Sun C, Fan L, Xu C, El-Toni AM, Alhoshan M, Zhang F. Anal Chem, 2018, 90: 7946-7952

70 Ning Y, Chen S, Chen H, Wang JX, He S, Liu YW, Cheng Z, Zhang JL. Inorg Chem Front, 2019, 6: 1962-1967

71 Sarder P, Maji D, Achilefu S. Bioconj Chem, 2015, 26: 963-974

72 Zhang KY, Yu Q, Wei H, Liu S, Zhao Q, Huang W. Chem Rev, 2018, 118: 1770-1839

73 Liao Z, Tropiano M, Faulkner S, Vosch T, Sørensen TJ. RSC Adv, 2015, 5: 70282-70286

74 Bui AT, Grichine A, Duperray A, Lidon P, Riobé F, Andraud C, Maury O. J Am Chem Soc, 2017, 139: 7693-7696

75 Ning Y, Cheng S, Wang JX, Liu YW, Feng W, Li F, Zhang JL. Chem Sci, 2019, 10: 4227-4235

76 Zhou Y, Chan CF, Kwong DWJ, Law GL, Cobb S, Wong WK, Wong KL. Chem Commun, 2017, 53: 557-560

77 Ning Y, Liu YW, Yang ZS, Yao Y, Kang L, Sessler JL, Zhang JL. J Am Chem Soc, 2020, 142: 6761-6768

\title{
Design and applications of NIR lanthanide molecular probes for bioimaging and biosensing
}

\author{
Xiao-Fei Zhu ${ }^{1,2 \dagger}$, Guo-Qing Jin ${ }^{1 \dagger}$, Jun-Long Zhang ${ }^{1 *}$ \\ ${ }^{1}$ Beijing National Laboratory for Molecular Sciences, State Key Laboratory of Rare Earth Materials Chemistry and Applications, College of \\ Chemistry and Molecular Engineering, Peking University, Beijing 100871, China \\ ${ }^{2}$ School of Chemistry and Life Science, Changchun University of Technology, Changchun 130012, China \\ $\dagger$ These authors contributed equally to this work. \\ *Corresponding author (email: zhangjunlong@pku.edu.cn)
}

\begin{abstract}
Near-infrared (NIR, 700-1700 nm) imaging has emerged as an advanced technology for real-time monitoring of various physiological and pathological procedures and visualization of biological entities. NIR imaging recently gains increasing attention owing to the less background interference, deeper tissue penetration, higher signal-to-noise ratio and temporal/spatial resolution. Furthermore, how to design NIR luminescent molecular probes becomes critical in biological NIR imaging. Lanthanide molecular probes have a promising prospective for the merits such as small size, low toxicity, long decay lifetime, low photobleaching and f-f transition of characteristic element emission. In this minireview, we summarized the sensitization principle and design strategy of NIR Ln molecular probes and the relevant biomedical applications.
\end{abstract}

Keywords: NIR lanthanide complexes, fluorescent probe, bioimaging, biosensing, lanthanide chemical biology

doi: $10.1360 /$ SSC-2020-0118 